\title{
Impact of patient-education on health related quality of life of diabetic foot ulcer patients: A randomized study
}

\author{
M. Sonal Sekhar ${ }^{\mathrm{a}, *}$, M.K. Unnikrishnan ${ }^{\mathrm{a}, 1}$, K. Vijayanarayana ${ }^{\mathrm{a}}$, Gabriel Sunil Rodrigues ${ }^{\mathrm{b}}$ \\ a Department of Pharmacy Practice, Manipal College of Pharmaceutical Sciences, Manipal Academy of Higher Education, Manipal, India \\ ${ }^{\mathrm{b}}$ Department of Surgery, Kasturba Medical College and Hospital, Manipal Academy of Higher Education, Manipal, India
}

\section{A R T I C L E I N F O}

\section{Keywords:}

Diabetic foot ulcer

Health-related quality of life

Patient-education

RAND-36

\begin{abstract}
A B S T R A C T
Background: Diabetic foot is the most serious and disabling long term complication of diabetes, adversely affecting the health related quality of life (HRQoL).

Objectives: To evaluates the impact of patient-education (PE) on HRQoL of diabetic foot ulcer (DFU) patients. Methods: This randomized controlled study on the effect of PE on improvement of HRQoL, conducted in Kasturba Hospital, Manipal, India consisted of 135 DFU patients with 65 subjects in the control group (CG) and 70 subjects in the intervention group (IG). RAND-36 questionnaire was employed for evaluating HRQoL scores of DFU patients in both groups at baseline and after six months of PE.

Results: Subjects in the both IG and CG reported poor HRQoL scores, on all the eight subscales at baseline. After six months of PE, HRQoL improved substantially in IG with respect to CG and IG at baseline (p $<0.05$ ). Likewise, in both CG and IG, the physical component summary scale (PCS) scores (24.1 \pm 6.6 vs $25.6 \pm 7.3)$ and the mental component summary scale (MCS) scores $(27.3 \pm 5.4$ vs $28.8 \pm 7.1)$ were similar and poor before PE. However, six months post PE, in IG, the Both PCS and MCS scores increased dramatically from $25.6 \pm 7.3$ to $42.9 \pm 9.7$ and $28.8 \pm 7.1$ to $48.8 \pm 8.4$ ( $\mathrm{p}<0.05)$ respectively.

Conclusion: The remarkable improvement in the HRQoL in IG at six months follow-up suggests that PE significantly improves HRQoL in DFU patients. Overworked physicians and paucity of trained doctors in resourcepoor settings obligate task-sharing with non-physician healthcare providers.
\end{abstract}

\section{Introduction}

Diabetic foot ulcer (DFU) is one of the most devastating complications of diabetes, which leads to suffering, disability, hospitalization, loss of productivity and high cost to the patient and society. ${ }^{1}$ Even in the developed countries, occurrence of DFU has been estimated to be about $8 \%$ and one in every 6 diabetics develop ulcer during their lifespan. ${ }^{2}$ Aggravation of DFU in India is further compounded by illiteracy, low socioeconomic status, inappropriate footwear, the practice of walking bare foot, lack of trained health professionals, near absence of patient counseling and ignorance in diabetic foot care. ${ }^{3}$

Health-professionals play a fundamental role in patient-education (PE) regarding foot hygiene, nail care and advice on proper footwear, which are absolutely necessary to reduce the risk of ulcer prone injury. Diabetic patients require education on daily foot care, awareness about issues that increase ulcer risk and when they should seek medical attention. There is reasonable evidence to prove that PE improves compliance and outcomes in DFUs. ${ }^{4}$ The importance of self-care and need for PE have been highlighted in a quantitative study (involving 4463 patient) by Lin et al., in $2004 .^{5}$ They reported that depression influences patients' behaviors and interferers with physical exercise, medication intake and foot care, while interaction between educators and patients improved outcomes. ${ }^{6}$

Many individuals with DFU experience contentment when they share their feelings with educators or care providers. Patient involvement is essential for the effective care of DFU. The fundamental duty of the health professional is to provide PE, self-assurance and support. Typically, $95 \%$ of care is administered by DFU patients and their families. $^{7}$ Therefore, educational modules to improve self-care are vital components of any effective management strategy.

DFUs have a major negative effect on health-related quality of life (HRQoL). ${ }^{8-11} \mathrm{DFU}$ is associated with reduced mobility and decline in

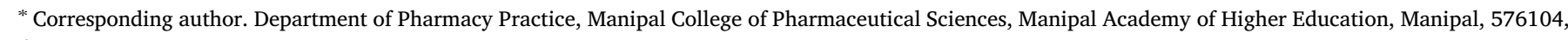
India.

E-mail addresses: sonalsekhar@gmail.com, sonal.sekhar@manipal.edu (M. Sonal Sekhar).

${ }^{1}$ Current affiliation: Principal, National College of Pharmacy, Kozhikode, Kerala, India.
} 
the daily activities, which can adversely affect HRQoL. A few studies indicate that day to day experiences of DFU patients without amputation may be even poorer than those who have undergone amputation. ${ }^{12}$ Addressing various aspects of the diabetic foot is perhaps best achieved by employing a multidisciplinary team to work through all stages of patient management. ${ }^{13} \mathrm{DFU}$ is a major burden for the individuals from the patient's immediate surroundings because it is the duty of caregivers to regularly support in wound care and cope with physical disabilities and emotional distress. Some studies show that patients with unhealed wounds were frustrated and anxious about healing, found it difficult to cope with daily living activities, inappropriate foot wear, and complained of a limited social life. ${ }^{14,15}$

Studies have shown that foot-education improves knowledge-level and satisfaction among high risk DFU patients. ${ }^{16}$ There is a lack of robust evidence to suggest that $\mathrm{PE}$ alone is effective in achieving improved HRQoL and clinically relevant reductions in incidence of DFU and amputation. ${ }^{17}$ In India, not many studies have been conducted to detect and analyze the HRQoL in DFU patients. Moreover, healthcare in India is dominantly guided by physicians, with suboptimal support from non-physician health professionals. ${ }^{18}$ Therefore we took up this study to assess the impact of PE on HRQoL of DFU patients in a tertiary care teaching hospital in South-India.

\section{Methods}

An open-labelled randomized controlled study was conducted in Kasturba Hospital (KH), Manipal, India after obtaining Institutional ethical clearance (Ref. No: IEC 410/2012).

\subsection{Study subjects}

Out of $260 \mathrm{DFU}$ patients admitted during the study period (January 2012 to December 2014), 210 were selected based on inclusion criteria and informed consent. They were randomly assigned into two groups (Intervention Group [IG] and Control Group [CG]) through envelop method of randomization. Of these, 135 patients completed follow-up (65 in CG and 70 in IG). The patients who were recruited for the IG were educated by the clinical pharmacists about various foot care measures and their importance by using patient information leaflets (PILs). PILs of either English or Kannada version was given to the patients, based on their choice. They were counselled about the importance of medication compliance, the need for off-loading, wound dressing, the use of properly fitting foot wear and also about annual podiatry reviews. Baseline HRQoL of both groups was assessed by using RAND-36 questionnaire, prior to educational intervention. Each patient was followed for a period of six months and HRQoL was re-assessed after six months.

\subsection{Patient interview}

Patient interview and counselling were carried out to create a favourable environment for acquiring data and providing education. Patients were interviewed during their free time in the hospital. After a direct interview of $20-25 \mathrm{~min}$, each patient was followed up by monthly telephonic contacts for ensuring continued motivation. Baseline HRQoL of both groups were measured, within two to three days of admission, using English or Kannada version of RAND-36 item health survey questionnaire. ${ }^{19}$

\subsection{Preparation of patient information leaflets}

PILs were modelled on various model leaflets from different online sources such as "Patient UK", Micromedex database etc. The content of leaflet was validated by the expert committee and appropriately revised. Readability of the designed DFU leaflet was checked online (www.readability-score.com) by calculating Flesch Readability Ease
(FRE) and Flesch-Kincaid Grade Level (FK-GL). Readability of the PIL was also assessed by user-testing method. Baker Able Leaflets Design (BALD) method was used to assess the layout and design characteristics of the PIL (Method published in Journal of Social Health and Diabetes (2013); 1 (2):79-81). ${ }^{20}$ Additionally, user-testing also carried out by quasi-experimental pre-and post-design evaluation of PILs within the IG with $34 \mathrm{DFU}$ patients for a period of 6 months (January 2012 to June 2012).

\subsection{Statistical methods}

Un-paired $t$-test was used to find the significance level within the controls and interventional groups. Paired $t$-test was used to assess the level of significance at follow-up. In this study $p<0.05$ is considered as statistically significant.

\subsection{Sample size}

The sample size of the study was calculated based on comparison of two population means. The required sample size per group is 61 . Sample size was calculated using nMaster software (Jeyaseelan et al., 2009) by using the following formula. ${ }^{21}$

$\mathrm{N}_{\text {pairs }}=\frac{\left(\mathrm{Z}_{1-\alpha / 2}+\mathrm{Z}_{1-\beta}\right)^{2}}{\Delta^{2}}+\frac{\mathrm{Z}_{1-\alpha / 2}^{2}}{2}$

$\Delta=\frac{\left(\mu_{2}-\mu_{1}\right)}{\sigma} \quad \sigma=\frac{\left(\sigma_{1}-\sigma_{2}\right)}{2}$

$=\frac{(1.96+0.84)^{2}}{(0.36)^{2}}+\frac{(1.96)^{2}}{2}=61$

Where,

Pre-test mean $\left(\mu_{1}\right)=10$

Post-test mean $\left(\mu_{2}\right)=12$

Standard deviation in the pre-test $\left(\sigma_{1}\right)=5$

Standard deviation in the post-test $\left(\sigma_{2}\right)=6$

Effect size $(\Delta)=0.36$

$Z_{1-\alpha / 2}$ for $\alpha(5 \%)=1.96$

$Z_{1-\beta}$ for $80 \%$ power $=0.84$

\section{Results}

The baseline characteristics of the study population are described in Table 1. Results show that both CG and IG were matched for demographic and clinical characteristics. Demographics of CG and IG show mean ( \pm SD) age is $60.3 \pm 8.4$ vs $58.6 \pm 7.9$. Majority of the patients in both groups were male ( $73.8 \%$ vs $78.6 \%)$. $44.6 \%$ of CG were employed as against $38.6 \%$ in the IG and disability was $18.5 \%$ in CG vs $21.4 \%$ IG. $78.3 \%$ of the CG and $71.7 \%$ of IG were from rural area. Majority of the DFU patients were T2DM in both CG and IG $(81.5 \%$ vs $78.6 \%)$ and were matched for HbA1c > 7.5 (66.2\% vs $61.4 \%)$. More than half of the study population in both groups had body mass index (BMI) between 23 and $27.5 \mathrm{~kg} / \mathrm{m}^{2}$. The mean duration of diabetes in both groups were $18.7 \pm 6.2$ vs $16.3 \pm 7.1$ years and duration of hospital stay was15.6 \pm 7.3 vs $18.5 \pm 6.4$ days.

More than half of the patients had ulcer size $<1 \mathrm{~cm}^{2}(63.1 \%$ vs $70.0 \%$ ). Majority of the patients in both groups had ulcers in the plantar digit region $(43.1 \%$ vs $51.4 \%)$. According to Wagner classification, most of the patients had superficial ulcers $(52.3 \%$ vs $60.0 \%)$ with polymicrobial growth $(47.7 \%$ vs $45.7 \%)$. Majority were on oral antidiabetic agents in both groups (78.5\% vs $71.4 \%)$.

\subsection{Health-related quality of life of diabetic foot ulcer patients at baseline}

Both CG as well as IG, at baseline, reported 'poor' HRQoL in all the subscales, especially physical functioning (PF) (16.5 \pm 5.6 vs $17.6 \pm 5.3$ ), role limitations due to physical health problems (RP) 
Table 1

Baseline characteristics of study population $(\mathrm{N}=135)$.

\begin{tabular}{|c|c|c|}
\hline \multirow[t]{2}{*}{ Parameters } & \multicolumn{2}{|l|}{ Values (\%) } \\
\hline & $C G(n=65)$ & IG $(n=70)$ \\
\hline Age (Mean $\pm S D$ ) years & $60.3 \pm 8.4$ & $58.6 \pm 7.9$ \\
\hline \multicolumn{3}{|l|}{ Gender } \\
\hline Male & $48(73.8)$ & $55(78.6)$ \\
\hline Female & $17(26.2)$ & $15(21.4)$ \\
\hline \multicolumn{3}{|l|}{ Employment } \\
\hline Working & $29(44.6)$ & $27(38.6)$ \\
\hline Disabled & $12(18.5)$ & $15(21.4)$ \\
\hline Retired & $24(36.9)$ & $28(40.0)$ \\
\hline \multicolumn{3}{|l|}{ Diabetes } \\
\hline Type 1 & $12(18.5)$ & $15(21.4)$ \\
\hline Type 2 & $53(81.5)$ & $55(78.6)$ \\
\hline \multicolumn{3}{|l|}{ Hb A1c (\%) } \\
\hline$<6.5$ & $07(10.7)$ & $13(18.6)$ \\
\hline $6.5-7.5$ & $15(23.1)$ & $14(20.0)$ \\
\hline$>7.5$ & $43(66.2)$ & $43(61.4)$ \\
\hline \multicolumn{3}{|l|}{ BMI $\left(\mathrm{Kg} / \mathrm{m}^{2}\right)$} \\
\hline $18.5-23$ & $23(35.4)$ & $29(41.4)$ \\
\hline $23-27.5$ & $34(52.3)$ & $39(55.7)$ \\
\hline$\geq 27.5$ & $08(12.3)$ & $02(02.9)$ \\
\hline Duration of diabetes (years) & $18.7 \pm 6.2$ & $16.3 \pm 7.1$ \\
\hline Duration of hospital stay (days) & $15.6 \pm 7.3$ & $18.5 \pm 6.4$ \\
\hline \multicolumn{3}{|l|}{ Ulcer size $\left(\mathrm{cm}^{2}\right)$} \\
\hline$<1$ & $41(63.1)$ & $49(70.0)$ \\
\hline $1-5$ & $20(30.8)$ & $19(27.1)$ \\
\hline$>5$ & $04(06.1)$ & $02(02.9)$ \\
\hline \multicolumn{3}{|l|}{ Ulcer location } \\
\hline Plantar digit & $28(43.1)$ & $36(51.4)$ \\
\hline Plantar metatarsal & $19(29.2)$ & $15(21.4)$ \\
\hline Dorsal digit & $13(20.0)$ & $12(17.2)$ \\
\hline Heel & $03(04.6)$ & $04(05.7)$ \\
\hline Lateral metatarsal & $02(03.1)$ & $03(04.3)$ \\
\hline \multicolumn{3}{|l|}{ Wagner's classification } \\
\hline Grade 1 & $34(52.3)$ & $42(60.0)$ \\
\hline Grade 2 & $12(18.5)$ & $10(14.3)$ \\
\hline Grade 3 & $16(24.6)$ & $13(18.6)$ \\
\hline Grade 4 & $03(04.6)$ & $05(07.1)$ \\
\hline \multicolumn{3}{|l|}{ Nature of microbial growth } \\
\hline No growth & $12(18.5)$ & $11(15.7)$ \\
\hline Monomicrobial & $22(33.8)$ & $27(38.6)$ \\
\hline Polymicrobial & $31(47.7)$ & $32(45.7)$ \\
\hline \multicolumn{3}{|l|}{ Treatment } \\
\hline Insulin & $40(61.5)$ & 39 (55.7) \\
\hline Oral antidiabetic agents & $51(78.4)$ & $50(71.4)$ \\
\hline
\end{tabular}

SD: Standard deviation.

$(7.7 \pm 3.7$ vs $9.5 \pm 4.6)$ and role limitations due to emotional health (RE) $(6.5 \pm 3.8$ vs $9.1 \pm 3.2)$ (Fig. 1). There is no significant difference between HRQoL under all the subscales between CG and IG at baseline $(p>0.05)$. Summary score of physical health (PCS) domains $(24.1 \pm 6.6$ vs $25.6 \pm 7.3)$ and mental health (MCS) domains $(27.3 \pm 5.4$ vs $28.8 \pm 7.1)$ were poor for DFU patients.

\subsection{Health-related quality of life of diabetic foot ulcer patients at six months follow-up}

Both CG and IG, at six month follow-up, report statistically significant differences $(p<0.05)$ in all the subscales of HRQoL (Fig. 2). HRQoL is 'better' in five subscales namely Energy/fatigue (EF), social functioning (SF), emotional well-being (EW), bodily pain (BP) and general health (GH)). Other three subscales (PF, RP and RE) score improved despite the very low mean scores $(<50)$. Results show that PE significantly improved mean PCS score $(26.5 \pm 5.1$ vs $42.9 \pm 9.7$, $p<0.05)$ and mean MCS score (30.1 \pm 7.4 vs $48.8 \pm 8.4, p<0.05)$ of HRQoL in DFU patients.

\subsection{Health-related quality of life of intervention group at baseline and follow up}

HRQoL of IG, between baseline and at six month follow-up, showed a significant difference $(p<0.05)$ in all the eight subscales (Fig. 3). HRQoL improved to 'better' in five subscales (EF, SF, EW, BP and GH). In the other three subscales (PF, RP and RE), HRQoL improved post interventionally, despite low mean score, $(<50)$ signifying 'poor' HRQoL. PE improved both mean PCS score (25.6 \pm 7.3 vs $42.9 \pm 9.7$, $p<0.05)$ and mean MCS score $(28.8 \pm 7.1$ vs $48.8 \pm 8.4, p<0.05)$ of HRQoL in DFU patients (Fig. 5).

\section{Discussion}

To the best of our knowledge, this is the first study to describe the highly significant impact of PE alone on HRQoL of DFU patients. Other educational intervention studies in the area of DFU were either focused on prevention of diabetic foot or averting amputation. ${ }^{17,22-25}$ Key findings of this study highlight the importance of the PE component for the improvement of HRQoL in DFU.

Baseline characteristics indicate that DFU is more prevalent among males and elderly populations. Male predominance in DFU could be linked to factors such as gender-related differences in life styles and professional roles that require the feet to tolerate more pressure. Increased level of outdoor work and poor compliance to foot care practices were more dominant among males than females. Another large retrospective cohort study also reported that prevalence of foot complications increased with age, mainly among male patients. ${ }^{26}$ Other studies (Jiang Y et al., 2015; Kalaivani (2014); Hadadi et al., 2014; and Ribu L et al., 2006) also reported similar observations. ${ }^{27-30}$ In our study, most of the DFU patients were working when included for the study. Ribu et al. (2007) reported similar findings, in which $40 \%$ of the patients were working. ${ }^{31}$ Majority of DFU patients in India are from rural areas, who earn their livelihood from labor intensive occupations. Mehra et al. (2008) reported high prevalence (10.4\%) of DFU in a hospital based rural Indian diabetic population. ${ }^{32}$

Majority of the DFU patents had T2DM, which is similar to reports by other research groups. ${ }^{26,31,33}$ In our study, patients with more than 15 years of diabetes developed DFU, which is similar to previous reports. ${ }^{26-30}$ Most patients in our study populations had HbA1c $>6.5 \%$, showing poor diabetic control in DFU. ${ }^{15,31,34}$ High BMI patients were at higher risk. ${ }^{15,} 30-34$

Most patients had ulcer size of $<1 \mathrm{~cm}^{2}$. This could be because our study included newly diagnosed diabetic foot patients with superficial ulcers. This is similar to the findings by others. ${ }^{30,31}$ However, studies from outside India by Hadadi et al. (2014) $)^{21}$ and Yekta et al. (2011) ${ }^{34}$ found that most of the patients had higher grade ulcers (deep ulcers). Most of our study subjects suffered from polymicrobial infections, which is similar to other reports from the abroad. ${ }^{35-37}$ However, Hadadi et al. (2014) reported higher prevalence of monomicrobial growth. ${ }^{29}$ Majority of our subjects were treated with oral antidiabetic agents, which is also similar to the findings of Yekta et al. (2011) study. ${ }^{34}$ However, Ribu et al. (2007) reported that most of the patients were on insulin. ${ }^{31}$

HRQoL is reported as mean scores, in which the 'average' score in a population is 50 . HRQoL is considered to be 'better', when the mean score is above 50. Both CG and IG, at baseline, reported 'poor' HRQoL in all the eight domains, which is similar to self-reported scores (in all the eight domains) of hospitalized with diabetic foot infection (DFI). ${ }^{8-15}$ Other studies have also reported significantly 'poor' HRQoL in all the 


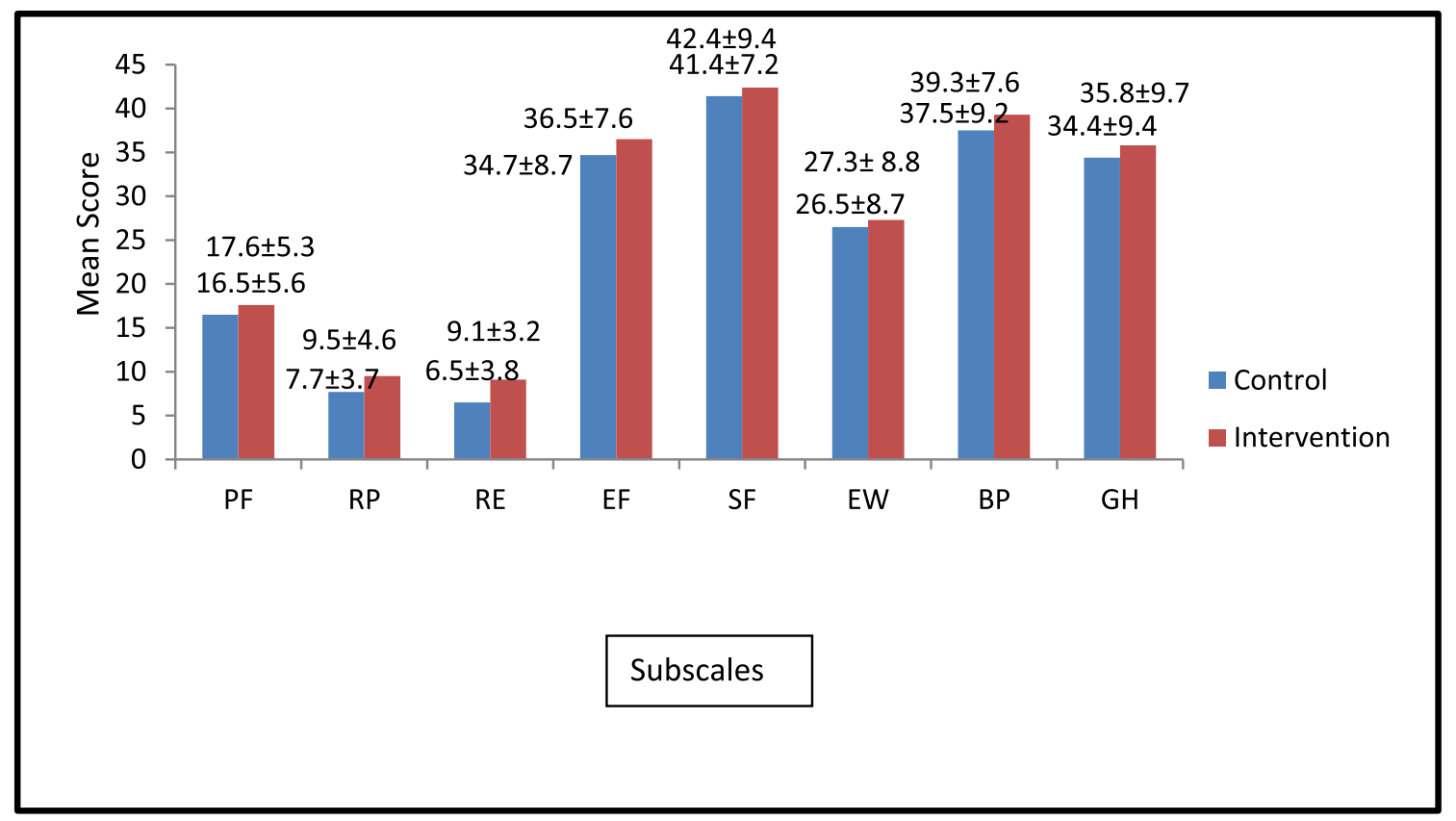

PF: Physical functioning; RP: Role limitations due to physical health; RE: Role limitations due to emotional health; EF: Energy/fatigue; SF: Social functioning;

EW: Emotional well-being; BP: Bodily pain; and GH: General health.

Fig. 1. Health-related quality of life of diabetic foot ulcer patients at baseline.

PF: Physical functioning; RP: Role limitations due to physical health; RE: Role limitations due to emotional health; EF: Energy/fatigue; SF: Social functioning; EW: Emotional well-being; BP: Bodily pain; and GH: General health.

subscale lines and summary scales of the patients with DFU, compared with both the general population and diabetic patients. ${ }^{38-40}$ This is because newly diagnosed DFUs were already in a poor condition when they first visited the podiatric clinic.

The three domains that were most severely compromised in both CG and IG, at baseline, were RE, RP and PF. This is supported by Mazline et al. (2011) ${ }^{39}$ and Ribu et al. (2007). ${ }^{15}$ Another study reported, in particular, lower values of + in DFU than in diabetics without foot ulcers. ${ }^{10}$ Similarly, significantly lower mean HRQoL in domains such as $\mathrm{PF}, \mathrm{RP}, \mathrm{SF}$ and RE have been previously reported. ${ }^{40}$ In our study, PCS and MCS were 'poor' for DFU patients. Both Raspovic et al. (2014) ${ }^{8}$ and Mazline et al. $(2011)^{39}$ have shown that PCS and MCS component scores were significantly decreased in DFI patients, denoting a negative effect on overall health. This is because DFUs have huge negative psychological and social effects, including decreased social activities, increased familial stress, limited employment and great financial hardship. Quantitative studies have confirmed these observations because DFUs exert a negative effect on physical, psychological and social states. $^{9}$

HRQoL improved to 'better' in five subscales (EF, SF, EW, BP and

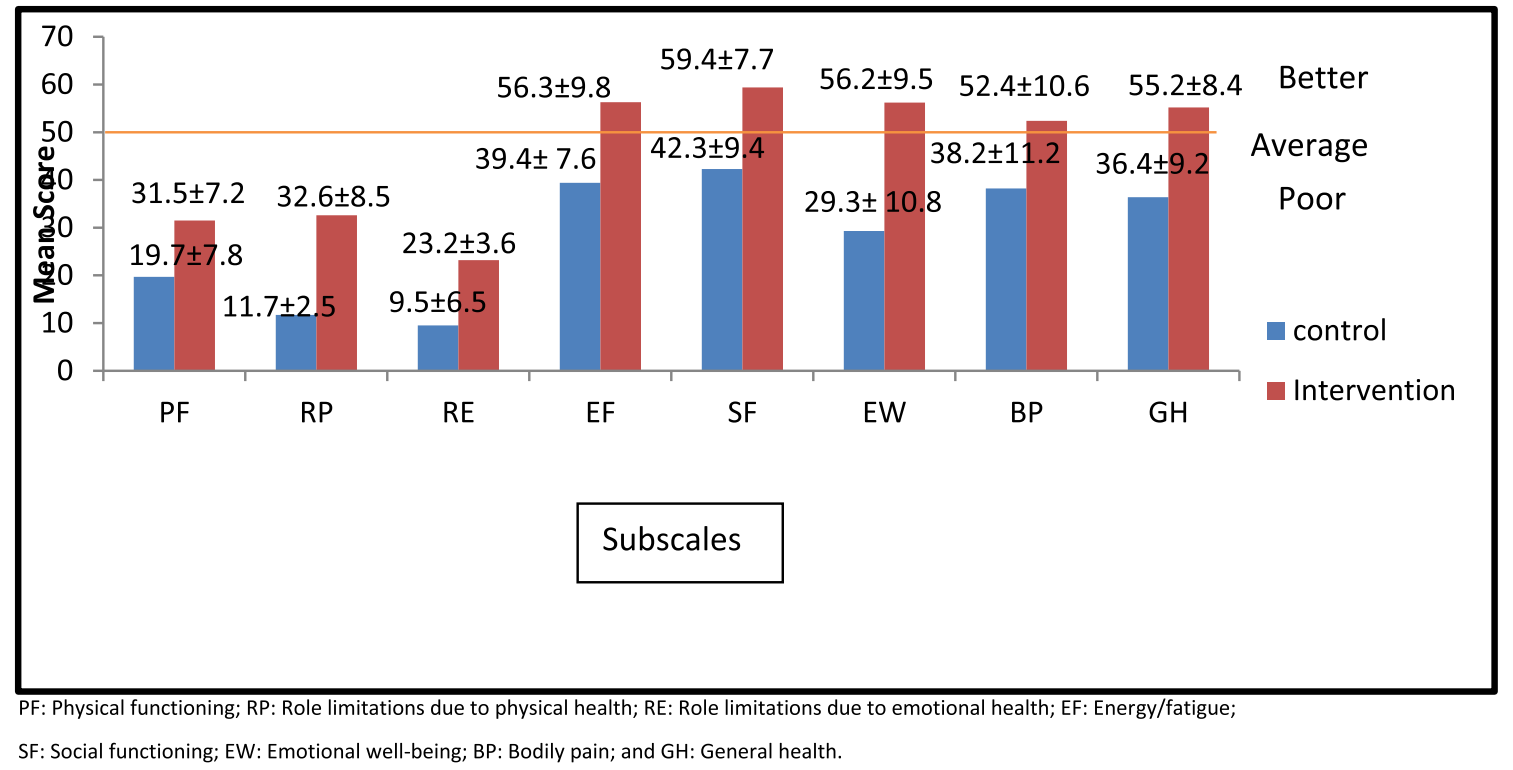

Fig. 2. Health-related quality of life DFU patients at six months follow-up.

PF: Physical functioning; RP: Role limitations due to physical health; RE: Role limitations due to emotional health; EF: Energy/fatigue; SF: Social functioning; EW: Emotional well-being; BP: Bodily pain; and GH: General health. 


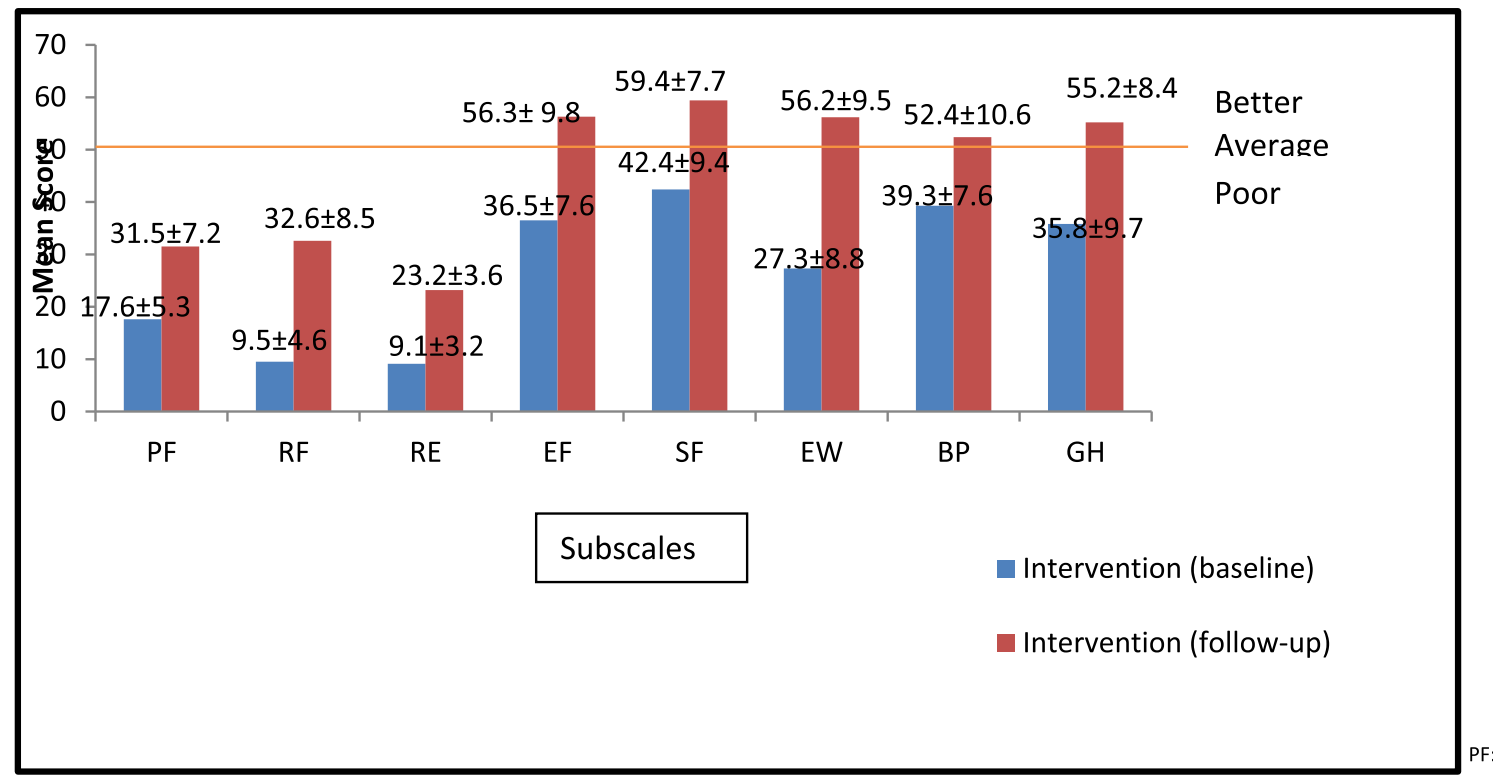

Physical functioning; RP: Role limitations due to physical health; RE: Role limitations due to emotional health; EF: Energy/fatigue; SF: Social functioning;

EW: Emotional well-being; BP: Bodily pain; and GH: General health.

Fig. 3. Health-related quality of life of IG at baseline and follow up.

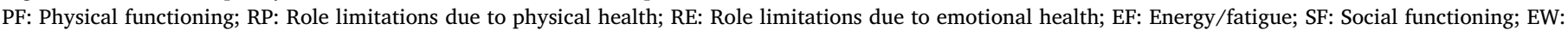
Emotional well-being; BP: Bodily pain; and GH: General health.

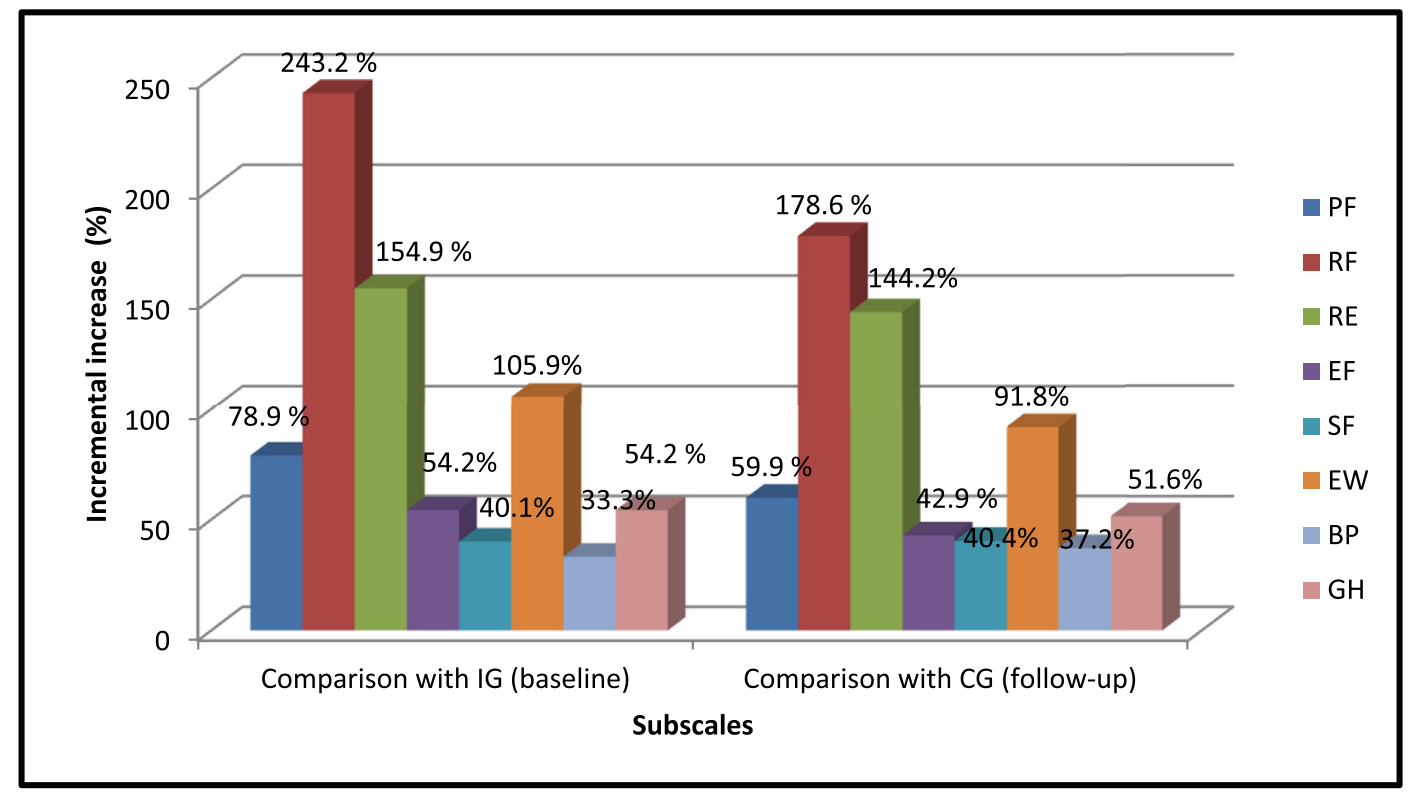

PF: Physical functioning; RP: Role limitations due to physical health; RE: Role limitations due to emotional health; EF: Energy/fatigue; SF: Social functioning;

EW: Emotional well-being; BP: Bodily pain; and GH: General health; IG: Intervention group; CG: Control group.

Fig. 4. Incremental increase in HRQoL subscales in IG at six month.

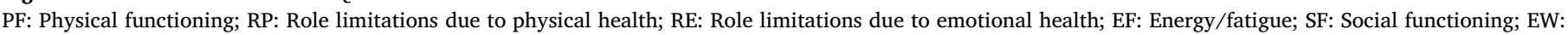
Emotional well-being; BP: Bodily pain; and GH: General health; IG: Intervention group; CG: Control group.

$\mathrm{GH}$ ) after educational intervention. Although the other three subscales (PF, RP and RE) improved dramatically by $78.9 \%, 243.2 \%$ and $154.9 \%$, respectively (Fig. 4). Yet, the scores still remained below 50, indicating 'poor' quality of life. This is because of the very low baseline scores in these domains. PE improved PCS and MCS scores of HRQoL in DFU patients, indicating improvement in both physical and mental health (Fig. 5). The above differences between IG and CG remained significant after adjusting for variables such as age, gender and duration of diabetes.
Our study has confirmed that PE is of paramount importance in improving HRQoL in DFU patients. This is partly supported by Yekta et al. (2011), in which they observed that a low educational level was associated with low HRQoL scores in DFU patients. ${ }^{34}$ Other studies also have shown that foot self-care education could improve knowledge and encourage regular foot care, thereby prevent ulcers.

HRQoL score is positively correlated to diabetes self-management status and glycemic control in DFU. Therefore, DFU patients should be motivated to perform self-management of their blood glucose. The 


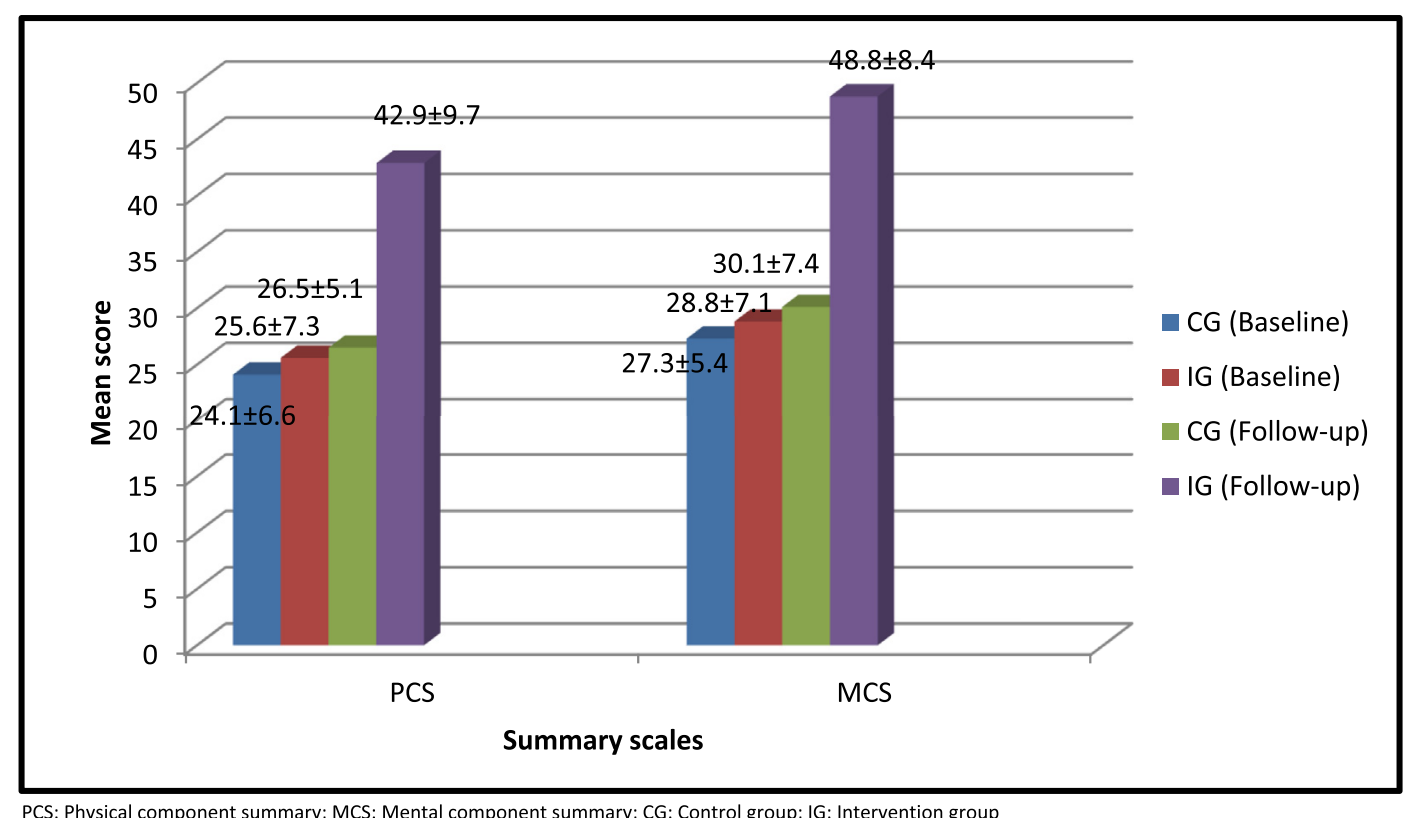

Fig. 5. Comparison of summary scores of HRQoL between CG and IG.

PCS: Physical component summary; MCS: Mental component summary; CG: Control group; IG: Intervention group.

strategies targeted at behavioral changes, such as PE, are effective for the metabolic control of the disease. Therefore, in order to improve HRQoL, treatment should not be focused exclusively on ulcer healing but also on multifactorial approaches that include health education.

Acute shortage of physicians, chiefly owing to extensive migration of trained professionals to developed Western countries, is an immense problem in India and shared by the developing world as a whole. ${ }^{18}$ The overworked medical practitioner appears to severely limit the implementation of quality healthcare delivery in India and many developing nations. Counseling the patient is either non-existent or ritualistic, and remains a low priority even in very prestigious hospitals. Our findings lay great emphasis on the need to develop an inclusive team of trained health professionals to support the physicians. 'Task-sharing' with non-physician health professionals like clinical pharmacists, besides being both feasible and less resource intensive, would go a long way in restricting the problem of DFUs, its associated vulnerabilities and consequent familial, social and economic impact. ${ }^{18}$

\section{Conclusion}

HRQoL in DFU is usually very poor without PE. We have observed a significant difference in all the subscales of HRQoL in the IG after six months. This improvement is more pronounced in domains such as $\mathrm{PF}$, $\mathrm{RP}, \mathrm{RE}$ and EW with an incremental surge of nearly twice or greater. Both physical and mental health domains, in DFU patients, improved with PE, demonstrating its value. We believe that educational intervention can improve adherence to both lifestyle modifications and medication that can improve HRQoL. Many DFU patients, predominantly manual laborers from lower socio-economic sections, live in the rural areas of resource-poor developing countries. The cost of imparting diabetic foot education would be more than compensated by the manifold benefits occasioned by improved HRQoL, resulting in increased labor productivity, lower treatment costs, shorter hospital stay, increased patient turnover, greater access to medical care and reduced DFU complications, including amputation. Such strategy is particularly appropriate for poorer countries like India, where health education and patient counseling are either completely ignored or minimally implemented. This study concludes that education per se by clinical pharmacists can significantly improve HRQoL. To the best of our knowledge, this is the first study that unequivocally confirms the superior impact of PE on HRQoL of DFU patients.

Overworked physicians and paucity of trained doctors in resourcepoor settings obligate task-sharing with non-physician healthcare providers. Educational intervention is an economical and feasible strategy, especially in the context of the ever-increasing burden of non-communicable disease in resource-poor developing world. Therefore, in order to improve HRQoL, management strategy should comprise multifactorial approaches that include health education.

\section{Conflicts of interest}

None.

\section{Source of funding}

Nil.

\section{Acknowledgement}

We express our gratitude to Manipal College of Pharmaceutical Sciences and Kasturba Medical College, Manipal Academy of Higher Education, Manipal for the support. Authors also acknowledge RAND for RAND-36 questionnaire.

\section{Appendix A. Supplementary data}

Supplementary data related to this article can be found at https:// doi.org/10.1016/j.cegh.2018.07.009.

\section{References}

1. Jeffcoate WJ, Harding KG. Diabetic foot ulcers. Lancet. 2003;361(9368):1545-1551. 2. Singh N, Armstrong DG, Lipsky BA. Preventing foot ulcers in patients with diabetes. $J$ Am Med Assoc. 2005;293(2):217-228.

3. Macfarlane RM, Jeffcoate WJ. Factors contributing to the presentation of diabetic foot ulcers. Diabet Med. 1997;14(10):867-870.

4. Bastable S. Nurse as Educator. second ed. Sudbury: Jones \& Bartlett; 2007.

5. Lin EH, Katon W, Von Korff M, et al. Relationship of depression and diabetes selfcare, medication adherence, and preventive care. Diabetes Care. 2004;27(9):2154-2160

6. Katon WJ, Von Korff M, Lin EH, et al. The Pathways Study: a randomized trial of collaborative care in patients with diabetes and depression. Arch Gen Psychiatr. 
2004;61(10):1042-1049.

7. Improving Supported Self-management for People with Diabetes. Diabetes UK; November 2009 https://www.diabetes.org.uk/Documents/Reports/Supported_selfmanagement.pdf Last accessed on September 2015.

8. Raspovic KM, Wukich DK. Self-reported quality of life and diabetic foot infections. $J$ Foot Ankle Surg. 2014;53(6):716-719.

9. Goodridge D, Trepman E, Embil JM. Health-related quality of life in diabetic patients with foot ulcers: literature review. J Wound, Ostomy Cont Nurs. 2005;32(6):368-377.

10. García-Morales E, Lázaro-Martínez JL, Martínez-Hernández D, Aragón-Sánchez J, Beneit-Montesinos JV, González-Jurado MA. Impact of diabetic foot related complications on the Health Related Quality of Life (HRQol) of patients-a regional study in Spain. Int J Low Extrem Wounds. 2011;10(1):6-11.

11. Sanjari M, Safari S, Shokoohi M, et al. A cross-sectional study in Kerman, Iran, on the effect of diabetic foot ulcer on health-related quality of life. Int $J$ Low Extrem Wounds. 2011;10(4):200-206.

12. Price $\mathrm{P}$, Harding $\mathrm{K}$. The impact of foot complications on health-related quality of life in patients with diabetes. J Cutan Med Surg. 2000;4(1):45-50.

13. Schaper NC, Apelqvist J, Bakker K. Reducing lower leg amputations in diabetes: a challenge for patients, healthcare providers and the healthcare system. Diabetologia 2012;55(7):1869-1872.

14. Ribu L, Birkeland K, Hanestad BR, Moum T, Rustoen T. A longitudinal study of patients with diabetes and foot ulcers and their health-related quality of life: wound healing and quality-of-life changes. $J$ Diabet Complicat. 2008;22(6):400-407.

15. Ribu L, Hanestad BR, Moum T, Birkeland K, Rustoen T. A comparison of the healthrelated quality of life in patients with diabetic foot ulcers, with a diabetes group and a nondiabetes group from the general population. Qual Life Res. 2007;16(2):179-189.

16. Ward A, Metz L, Oddone EZ, Edelman D. Foot education improves knowledge and satisfaction among patients at high risk for diabetic foot ulcer. Diabetes Educat. 1999;25(4):560-567.

17. Dorresteijn JA, Kriegsman DM, Assendelft WJ, Valk GD. Patient education for preventing diabetic foot ulceration. Cochrane Database Syst Rev. 2014;12:CD001488.

18. Sharma A, Ladd E, Unnikrishnan MK. Healthcare inequity and physician scarcity empowering non-physician healthcare. Econ Polit Wkly. 2013;XLVIII(13):112-117.

19. Hays RD, Morales LS. The RAND-36 measure of health-related quality of life. Santa Monica, California: RAND Corporation, RP-971; 2001 As of April 17, 2015 http:// www.rand.org/pubs/reprints/RP971.

20. Roy RT, Sekhar MS, Rodrigues GS, Rajesh V. Preparation and readability assessment of patient information leaflets for diabetic foot ulcers. $J$ Soc Health Diabetes. 2013;1(2):79-81.

21. Jeyaseelan L, Neelakantan N, Balakumar R, Jeyaseelan V, Kumar VS. nMaster V 2.0. Vellore: Christian Medical College; 2009.

22. Dorresteijn JA, Valk GD. Patient education for preventing diabetic foot ulceration. Diabetes Metab Res Rev. 2012;28(Suppl 1):101-106.

23. Valk GD, Kriegsman DM, Assendelft WJ. Patient education for preventing diabetic foot ulceration. A systematic review. Endocrinol Metab Clin N Am 2002;31(3):633-658.
24. Boulton AJ. Why bother educating the multi-disciplinary team and the patient-the example of prevention of lower extremity amputation in diabetes. Patient Educ Counsel. 1995;26(1-3):183-188.

25. Apelqvist J, Larsson J. What is the most effective way to reduce incidence of amputation in the diabetic foot? Diabetes Metab Res Rev. 2000;16(Suppl 1):S75-S83.

26. Al-Rubeaan K, Al Derwish M, Ouizi S, et al. Diabetic foot complications and their risk factors from a large retrospective cohort study. PLoS One. 2015;10(5):e0124446.

27. Jiang Y, Ran X, Jia L, et al. Epidemiology of type 2 diabetic foot problems and predictive factors for amputation in China. Int $J$ Low Extrem Wounds. 2015;14(1):19-27.

28. Kalaivani V. Evaluation of diabetic foot complications according to amit jain's classification. J Clin Diagn Res. 2014;8(12):NC07-9.

29. Hadadi A, Omdeh Ghiasi H, Hajiabdolbaghi M, Zandekarimi M, Hamidian R. Diabetic foot: infections and outcomes in Iranian admitted patients. Jundishapur $J$ Microbiol. 2014;7(7):e11680.

30. Ribu L, Rustøen T, Birkeland K, Hanestad BR, Paul SM, Miaskowski C. The prevalence and occurrence of diabetic foot ulcer pain and its impact on health-related quality of life. J Pain. 2006;7(4):290-299.

31. Ribu L, Hanestad BR, Moum T, Birkeland K, Rustoen T. Health-related quality of life among patients with diabetes and foot ulcers: association with demographic and clinical characteristics. J Diabet Complicat. 2007;21(4):227-236.

32. Mehra BR, Thawait AP, Karandikar SS, Gupta DO, Narang RR. Evaluation of foot problems among diabetics in rural population. Indian J Surg. 2008;70(4):175-180.

33. Assaad-Khalil SH, Zaki A, Rehim AA, et al. Prevalence of diabetic foot disorders and related risk factors among Egyptian subjects with diabetes. Prim Care Diabetes. 2015;9(4):297-303.

34. Yekta Z, Pourali R, Ghasemi-Rad M. Comparison of demographic and clinical characteristics influencing health-related quality of life in patients with diabetic foot ulcers and those without foot ulcers. Diabetes Metab Syndr Obes. 2011;4:393-399.

35. Lipsky BA, Berendt AR, Cornia PB, et al. Infectious diseases society of America clinical practice guideline for the diagnosis and treatment of diabetic foot infections. J Am Podiatr Med Assoc. 2013. 2012;103(1):2-7.

36. Radji M, Putri CS, Fauziyah S. Antibiotic therapy for diabetic foot infections in a tertiary care hospital in Jakarta, Indonesia. Diabetes Metab Syndr. 2014;8(4):221-224.

37. Al Benwan K, Al Mulla A, Rotimi VO. A study of the microbiology of diabetic foot infections in a teaching hospital in Kuwait. J Infect Public Health. 2012:5(1):1-8.

38. Yao H, Ting X, Minjie W, et al. The investigation of demographic characteristics and the health-related quality of life in patients with diabetic foot ulcers at first presentation. Int J Low Extrem Wounds. 2012;11(3):187-193.

39. Mazlina M, Shamsul AS, Jeffery FA. Health-related quality of life in patients with diabetic foot problems in Malaysia. Med J Malaysia. 2011;66(3):234-238.

40. de Meneses LC, Blanes L, Francescato Veiga D, Carvalho Gomes H, Masako Ferreira L Health-related quality of life and self-esteem in patients with diabetic foot ulcers: results of a cross-sectional comparative study. Ostomy/Wound Manas. 2011;57(3):36-43 